\title{
Visual selection and response selection without effector selection in tasks with circular arrays
}

\author{
Robert W. Proctor $^{1}$ (D) $\cdot$ Alice F. Healy ${ }^{2,3}$ \\ Published online: 9 September 2020 \\ (C) The Psychonomic Society, Inc. 2020
}

\begin{abstract}
Charles Eriksen and colleagues conducted influential visual-search experiments with circular arrays for which the responses were either vocal naming or unimanual left-right switch movements. These methods have the advantages of the stimuli being equidistant from a centered fixation point and allowing study of visual selection and response selection when effector selection is not required, as in the more typical case in which responses are key presses of distinct fingers. Other researchers have used similar spatial arrangements, but with aimed movements of the limb or of a mouse-controlled cursor to study effects of stimulus identification, visual search, spatial stimulus-response compatibility, response-effect compatibility, and practice/transfer in isolation and jointly. We systematically review studies in these areas that include visual selection and response selection and execution, and examine implications of their results for the role of effector selection. Also, we illustrate that as one moves from simpler to more complex tasks, the results are consistent with a basic information-processing framework in which stimulus identification and selection of a target response location are distinct from selecting, planning, and moving an effector to the targeted location.
\end{abstract}

Keywords Attention: Selective · Goal-directed movements · Perception and Action · Response-effect compatibility · Spatial information processing $\cdot$ Stimulus-response compatibility $\cdot$ Visual search

\section{Prelude}

When we think of Charles W. Eriksen's research on spatiotemporal characteristics of visual attention and information processing, incisive, logically, and empirically precise studies come to mind. He was meticulous in his investigations of such topics as visual search, spatial cuing, and correspondence effects of flanking stimuli on an attended target (e.g., C. W. Eriksen \& Hoffman, 1973). His research used multiple converging operations, as advocated in the classic article by Garner, Hake, and Eriksen (1956), to isolate, identify, and

Robert W. Proctor

rproctor@purdue.edu

1 Department of Psychological Sciences, Purdue University, 703 Third St., West Lafayette, IN 47907, USA

2 Department of Psychology and Neuroscience, University of Colorado Boulder, Boulder, CO 80309-0345, USA

3 Present address: 1901 Walnut Street, Apt 16A, Philadelphia, PA 19103, USA confirm the processes underlying performance of visual information-processing tasks. Much of Eriksen's research used circular arrays to study visual attention and unimanual responses that did not require effector selection. These methods and their extensions allow investigation of a range of spatial factors in human information processing without complication from nonspatial factors such as differences in retinal sensitivity and selection of a response effector, as occurs when stimuli are various distances from the fovea and responses are key presses made with distinct fingers.

In this article, we offer a detailed review of spatial information processing studies using such arrays and responses, starting with the work of Eriksen and colleagues on selective attention and target identification in visual displays. We then progress to studies examining stimulus-response compatibility and uncertainty effects in response selection, continue with studies investigating visual search for a specified targetlabeled location, and end with experiments that isolate influences of response-effect compatibility on performance. This review takes us far afield from the investigations of Eriksen and colleagues, but we see the reported analytic research of visual information processing as inspired by their work and in the same spirit. 


\section{Overview and goal}

Visual selective attention and response selection are research areas that have been prominent over the past several decades (Hommel, Brown, \& Nattkemper, 2016; Xiong \& Proctor, 2018). They are usually studied separately but are closely related, as one requires selection from alternative display items and the other selection from alternative responses. One topic of interest is the extent to which attention can be focused on a specific location for responding to a target without interference from flanking stimuli (Wühr \& Heuer, 2017). Another is compatibility effects of various types (Proctor \& Vu, 2016). These effects consist of stimulus-response compatibility for mappings of relevant stimulus dimensions to responses, for which certain mappings yield better performance than others (Proctor \& Vu, 2016), and response-effect compatibility for mappings of responses to effects that they produce (Janczyk \& Lerche, 2019). Compatibility effects also include those for correspondence of irrelevant stimulus dimensions with responses, in which a nominally irrelevant stimulus feature yields better performance when it corresponds with the response (Luo \& Proctor, 2020), and correspondence of irrelevant and relevant stimulus dimensions (Verbruggen, Liefooghe, Notebaert, \& Vandierendonck, 2005). Many visual attention and response selection studies have used twochoice tasks for which left and right key-press responses are made with the index fingers of the respective hands and reaction time is measured. Although those studies are valuable and informative for many purposes (e.g., Bravo \& Nakayama, 1992; Proctor \& Cho, 2006), much can be learned from examining more complex situations.

Of particular interest are tasks in which participants make unimanual aimed movements of a finger, stylus, or cursor to a target location in response to one of several possible stimuli. Fitts and Seeger (1953), in their classic study of stimulus-response compatibility, described benefits of using aimed-movement responses in terms of the concept of a response code. In their words, "A unidimensional response code utilizes a single effector member and a specified number of points along one of the physical dimensions of a particular response continuum, such as the direction, the force, or the duration of a movement" (p. 200). As emphasized recently by Wright, Marino, Chubb, and Mann (2019), such situations allow isolation of response-selection processes at a level of spatial locations without including the level of effector selection, as required for key-press responses. Because Wright et al. (2019) have made a strong case that effector selection is a distinct process that may affect results obtained in choice-reaction tasks, we focus mainly on tasks where selection of an effector is not an issue. A reason for doing so is that any phenomenon evident in such tasks cannot be attributed to effector selection.
Aimed-movement responses also allow partitioning of factors that influence time to initiate a signaled response (initiation time; IT) from those that affect time between initiation of the movement and contact with the target response location (movement time; MT). IT is sometimes called reaction time (and the sum of IT and MT, total response time), but in this article we restrict use of reaction time to discrete key-press, vocal, and lever-switch push responses. Also, with aimedmovement responses, influences of both stimulus-response and response-effect compatibility can be examined, as can transfer across systematically altered conditions.

Therefore, in the present article, we review research conducted mainly with unimanual-movement responses, beginning with visual-search studies by Eriksen and colleagues for which the imperative stimulus is a to-be-identified target letter at a cued location in a circular array. This topic is followed by studies of response selection in which the relevant stimulus is the location at which a stimulus occurs, which requires a preassigned response in a similar, circular response array. We then proceed through studies of more complex tasks that entail identification of alphanumeric stimuli at a designated location or matching of a target stimulus to a labeled location, and end with studies in which the mapping of control movements to a controlled cursor is varied. The goal is to analyze and categorize the influences of various factors on visual selection and response selection that are independent of effector selection.

\section{Visual selection of a target alphanumeric character at a cued location}

Eriksen and colleagues conducted several relevant visualsearch studies in which the circular locations were occupied by letter stimuli, among which was a target stimulus at a cued location, to which a response was to be made. These studies used vocal naming or unimanual left-right switches of a lever, and hence did not require effector selection. We consider here only those for which response time, not response accuracy, was the primary dependent measure.

\section{Visual search with vocal identification responses}

C. W. Eriksen and Hoffman (1972a) had participants respond by naming as quickly as possible a target letter in an array of four, eight, or 12 letters (composed of the possible targets A, $\mathrm{H}$, T, and U; see Fig. 1). The letters in the largest array were positioned at the locations corresponding to the 12 positions on a clock face. For the eight-letter displays, letters were presented at the 12,3,6, and 9 o'clock positions (vertical and horizontal axis locations), and the remaining four letters were located midway between those positions. For the four-letter displays, the letters were in the four midway positions (i.e., 


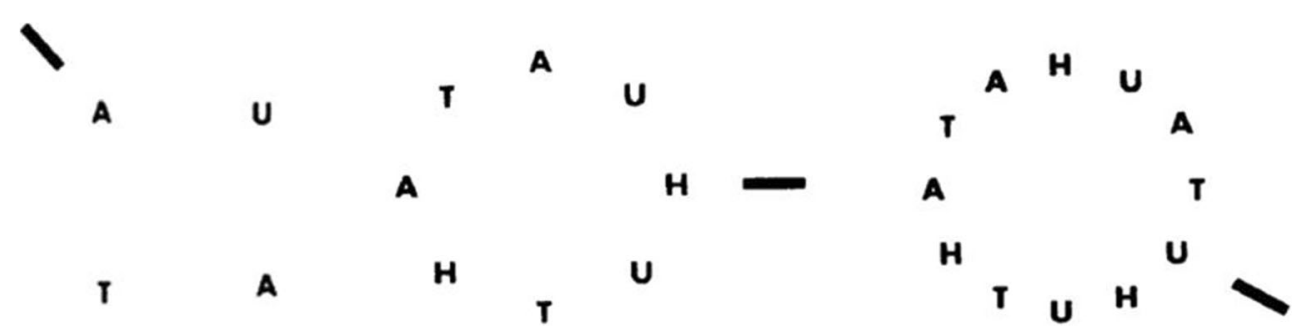

Fig. 1 Example four-letter, eight-letter, and 12-letter displays used by C. W. Eriksen and Hoffman (1972a), with bar marker indicators shown

1:30, 4:30, 7:30, 10:30). The target stimulus was signaled by a black bar outside of the array "on an extension of an imaginary radius from the center of the display through the indicated position" (p. 11) such that it pointed to the letter that was to be reported. The bar onset was either simultaneous with that of the array or 150-ms before it.

The primary results were that vocal reaction time increased progressively as display size increased, and was shorter when the black bar preceded the array by $150 \mathrm{~ms}$, implying that this lead time was sufficient for participants to direct attention to the target location. Because the benefit of precuing the location was greater for the two larger array sizes than for the smallest one, the difference in reaction time between the 12item and four-item arrays was reduced from $70 \mathrm{~ms}$ without the lead time to $36 \mathrm{~ms}$ with it. Of interest, when the bar indicator preceded the array onset, mean reaction time was only $10 \mathrm{~ms}$ longer for the eight-item array than for the four-item array.

The experiments by C. W. Eriksen and Hoffman (1972b) used five-element displays, for which the target letter was located in one of the 12 clock positions, with two noise stimuli on each side. Spacing between the target and noise was varied, with zero, one, or two blank clock positions between the target and noise stimuli. The noise could be instances of the four possible target letters (A, H, M, and U) or black disks of the same size as the letters. The black bar position indicator always onset simultaneously with the target letter, and the noise elements appeared at stimulus onset asynchronies (SOAs) of 0 , $50,100,150,200$, and $300 \mathrm{~ms}$. At 0-ms SOA, the noise letters produce much longer reaction times than did the noise disks. Also, letters in the adjacent positions produced more interference than those separated from the target by one or two spaces, for which the reaction times did not differ. This result implies that participants were able to direct attention to an area that included not only the target but locations to either side. The differences between all the noise conditions were absent when the target onset preceded that of the noise by $150 \mathrm{~ms}$ or longer. C. W. Eriksen and Hoffman (1972b) concluded that their results were in agreement with the view that the focus of attention subtends approximately $1^{\circ}$ of visual angle.

Colegate, Hoffman, and Eriksen (1973) compared reaction times for displays of eight and 12 letters, with the indicator preceding the array onset by an SOA of $0,50,100,150$, or $250 \mathrm{~ms}$ in their Experiment 1. In a control condition, a neutral warning (a black dot in the center of the display) was presented at one of the SOAs before array onset, but with the bar indicator appearing simultaneously with the display. There was an effect of display size, with reaction time $30 \mathrm{~ms}$ longer for the 12-letter arrays than for the eight-letter arrays, and responses were faster with the bar indicator compared with the neutral warning, with this difference increasing as SOA increased. For the bar indicator, throughout the range of SOAs up to $150 \mathrm{~ms}$, the reaction-time difference between the two display sizes stayed the same, with a slight tendency for the 12-letter condition to converge toward the eight-letter condition at the 250-ms SOA. Experiment 2 extended the longest SOA to $350 \mathrm{~ms}$ and found that the difference between reaction times for the 12-letter and eight-letter arrays remained at the long SOA. In addition, eye movements were recorded, and it was concluded that the reaction-time results could not be attributed to eye movements, but were due to a central encoding mechanism "limited in its precision of localization and exclusion" (p. 217). That is, the mechanism is not able to focus solely on the target location and exclude near letters.

\section{Visual selection with unimanual switch-movement responses}

C. W. Eriksen and Hoffman (1973) examined two-choice manual reactions, as more typically used in reaction-time studies, but with the responses made using a spring-loaded lever switch mounted on the right arm of a hand rest. The switch was held between the thumb and index finger of the right hand. Specifically, they used 12-letter displays, composed from the letters A, H, M, and U. Participants were to respond with a lever movement in one direction (left or right) if the target letter designated by the black bar indicator was A or U, and in the other direction if it was $\mathrm{H}$ or M. Independent variables were the SOA by which the bar indicator preceded onset of the array $(0,50,150$, and $250 \mathrm{~ms}$ in one experiment; $0,50,150$, and $350 \mathrm{~ms}$ in another) and whether letters adjacent to the target were from the same or opposite set.

With simultaneous onset of the bar indicator and array, reaction time was much longer when the flanking letters were members of the set assigned to the opposite response from the target than when they were of the same set, a finding known as the flanker compatibility effect. This result implies that the interference produced by incongruent noise letters is chiefly on response selection rather than letter identification. The interference 
of opposite-set letters was less when they were more distant from the target in the array, implying a limit to the precision of selective visual attention. These latter two findings were confirmed in the classic flanker task of B. A. Eriksen and Eriksen (1974) and C. W. Eriksen and Eriksen (1979), for which search was not necessary because the target was presented at a constant, centered location, yet reaction time was still prolonged by incongruent noise stimuli in close spatial proximity.

An experiment of C. W. Eriksen and St. James (1986) had the unique aspect of cuing one, two, or three adjacent positions of an eight-letter circular display. One of two possible target letters occurred randomly in one of the cued positions, and a congruent or incongruent noise letter could occur one, two, or three positions outside of the edge of the cued area. Reaction times to classify the target letter lengthened as the number of cued locations increased from one to three, suggesting reduced processing efficiency with a larger region of attentional focus. However, the interference produced by an incongruent noise letter outside of the cued area was independent of the number of cued locations. To be able to present an incongruent letter inside as well as outside of attentional focus, the noise letters in Experiment 2 were visually similar to the targets (targets were $\mathrm{N}$ and $\mathrm{Y}$, and incongruent noise letters were $\mathrm{H}$ and $\mathrm{V}$ ). When two positions were cued, a noise letter produced more interference if it was in the adjacent cued location than if it was in the adjacent uncued location. C. W. Eriksen and St. James interpreted their results in terms of a zoom lens model, according to which people strategically adjust the size of the attentional focus to the cued region, with a gradual transition at the edge. This model is still considered to capture a broad range of results in visual selective attention (Cave \& Chen, 2016).

Descriptions of the flanker task often overlook that all of these studies by Eriksen and colleagues showing the flanker compatibility effect used left-right lever-press responses. A consequence of this methodological factor, whether intended or not, is that, at a minimum, the interference from incongruent flankers is not dependent on the requirement to select an effector. One reason why this fact is essential to keep in mind is that widely cited studies by Coles, Gratton, Bashore, Eriksen, and Donchin (1985) and Gratton, Coles, Sirevaag, Eriksen, and Donchin (1988) can be misunderstood as establishing a basis for the flanker effect in effector selection. In those studies, participants responded to the $\mathrm{H}$ and $\mathrm{S}$ letter stimuli with lefthand and right-hand dynamometer squeezes, and measures of motor activation (including lateralized readiness cortical potentials and electromyograms) were recorded in addition to reaction time. Results showed standard flanker compatibility effects, and the various measures provided indications on incongruent trials of activation of the incorrect response hand.

Coles et al. (1985) and Gratton et al. (1988) discussed their results in terms of response channels, of which Gratton et al. said, "We use this term as a heuristic device to refer to that complex of structures whose activities are more or less directly related to the mechanical event that is defined as the overt response" (p. 331). As Mattler (2005) noted, "A peripheral locus of response competition is suggested by this concept, because it relates response activation more or less directly to electrophysiological measures of motor activation" ( $\mathrm{p}$. 579). Yet, the fact that the flanker effect occurs with unimanual switch movements indicates that it cannot possibly be based entirely in effector selection. Mattler conducted a study modeled after those of Coles et al. and Gratton et al. in which he analyzed the relation between reaction time and various response activation measures. He reached an even stronger conclusion: "Results suggested that the flanker compatibility effect cannot be attributed to peripheral processes of response activation, which are reflected in measures of the overt motor output" (p. 599, emphasis added).

\section{Empirical summary}

1. Presentation of a bar cue $150 \mathrm{~ms}$ or longer prior to onset of the letter array reduces reaction time, implying an ability to shift visual attention to the cued location.

2. Number of noise elements in the display and their spacing relative to the target letter both influence reaction time to identify the target.

3. Spacing between adjacent elements has an effect primarily when the noise stimuli are within $1^{\circ}$ of visual angle of the target letter, indicating a limit of selective attention.

4. The attentional focus can be expanded to include more than one location, if needed for the task.

5. The flanking noise stimuli produce interference mainly when they signal the opposite response of that assigned to the target, providing evidence of response competition.

6. This interference is eliminated when target onset precedes the noise letters by $150 \mathrm{~ms}$.

7. This flanker effect occurs with naming and unimanual lever-press responses, which do not require effector selection, indicating that it is not based exclusively, or perhaps even partially, in processes involved in selecting an effector with which to respond.

\section{Response selection with circular stimulus and response arrays}

In their articles introducing the concept of stimulus-response compatibility, Fitts and colleagues (Fitts \& Deininger, 1954; Fitts \& Seeger, 1953) used tasks for which the alternative stimulus locations signaled target locations to which a movement response was to be made. They were interested primarily in performance with various pairings of display and response configurations and mappings of individual stimulus elements to response elements. The more recent work by Wright and 
colleagues (e.g., Wright et al., 2019) has focused on the influence of uncertainty, or set size - that is, the number of possible stimulus-response alternatives.

\section{Stimulus-response compatibility}

Fitts and Seeger (1953) compared performance when participants moved one or two styli down paths emanating from a centered starting position to designate the corresponding location in a response array to that of one of eight light stimuli that occurred on a display panel. The study used three stimulus arrays - eight circular locations (Set A); four horizontal and vertical locations (Set B), singly or in pairs to designate diagonal locations (Set $\mathrm{C}$ ); and two horizontal locations separate from two vertical locations, again singly or in pairs. These were paired with three response arrays - two of which required unimanual movements (paths radiating outward to eight circular-arrayed locations; horizontal and vertical paths requiring movements in each to reach diagonal locations), and one of which required bimanual movements (separate horizontal and vertical paths, operated by styli in the left and right hands, respectively). Mean IT was less and errors fewer when the stimulus and response arrays matched than when they did not, and IT was numerically shortest for the circular stimulus and response arrays.

In a second experiment, five participants performed for 32 sessions each using the three stimulus arrays mapped to the circular response array, practicing each combination 16 times per session. Set A (the circular stimulus array that corresponded with the circular response array) yielded the shortest IT throughout the experiment. In Sessions 17-26, prior to introduction of a secondary distracting task on Day 27, mean IT for stimulus Sets A, B, and C, respectively, was 272,286 , and $355 \mathrm{~ms}$ (with accompanying error rates of $6.9 \%$, $10.0 \%$, and $11.9 \%$ ). Inclusion of a concurrent secondary mental arithmetic task in Sessions 27-30 increased IT to $400 \mathrm{~ms}$ for all conditions (estimated from Fitts \& Seeger's, 1953, Fig. 4). Accordingly, the cost in IT for Condition C compared with Condition A was reduced from $83 \mathrm{~ms}$ in Sessions 17-26 to 20 $\mathrm{ms}$. In contrast, the difference in error rates between Conditions $\mathrm{C}$ and $\mathrm{A}(4.4 \%, 6.9 \%$, and $15.6 \%$ for Conditions A, B, and C) increased compared with Sessions 17-26 (which, again, were $6.9 \%, 10.0 \%$, and $11.9 \%$, respectively). Fitts and Seeger interpreted these results as "the introduction of an additional task late in learning in this case apparently served to reduce the degree of readiness for the motor task, minimizing reaction-time differences, but increasing differences with respect to errors in the motor task" (p. 208), but they noted that the data were inconclusive.

MT was recorded for Fitts and Seeger's (1953) Experiment 2 , but reported only with the brief description that in Sessions 17 through 26 it was slightly longer for Set C $(67 \mathrm{~ms})$ than for Sets A and B (59 and $61 \mathrm{~ms}$, respectively). The short MT may be because the stylus was moved in a track from the centered initial position to a terminal target location (requiring no deceleration since the stylus would reach a stop). The small carryover of the differences between conditions into MT likely still reflects response selection, because the instructions emphasized responding quickly rather than beginning the movement only after the response was identified.

Of more interest for present purposes, Fitts and Deininger (1954) used the eight-choice circular arrays for both stimuli and responses, but varied the mapping between individual locations. In one condition, participants were to move to the response location that corresponded to the stimulus location. In a second condition, the mapping of stimulus-response locations was random. Not surprisingly, IT and error rate were much lower in the first condition (387 ms; $1.9 \%$ ) than in the second one $(1,111 \mathrm{~ms} ; 15.1 \%)$. In a third condition, the relation between stimulus and response locations was left-right mirror reversal. In this case, performance (541 ms; $4.4 \%$ ) was not as good as with the corresponding mapping, but was much better than with the random mapping, indicating that participants could use a "respond mirror opposite" rule to facilitate response selection. Morin and Grant (1955) likewise found that for an eight-choice key-press task with linear arrays a mirror-reversed mapping yielded considerably slower responses than a direct mapping, but not nearly as slow as mappings for which the stimulus and response locations had little or no correlation (see also Chen \& Proctor, 2015).

Fitts and Deininger (1954) also investigated performance with a symbolic stimulus set mapped to the same circular response set. The stimulus set was eight three-digit and fourdigit numbers that corresponded to the eight response locations on a clock face (12:00, 1:30, 3:00, etc.). Responses were again faster and had a lower error rate $(675 \mathrm{~ms} ; 5.0 \%)$ for a condition in which the mapping to response locations corresponded with the stereotypical relation for a standard clock face (12:00 designated top position, 1:30 the first diagonal position clockwise from the top, 3:00 the right response position, etc.) compared with a condition in which the mapping was random (885 ms; $10.0 \%)$. In addition, a condition with mirror-reversed mapping (e.g., 1:30 designated the first diagonal position counterclockwise from the top; 3:00 designated the left response position) showed intermediate IT and error rate $(777 \mathrm{~ms} ; 7.2 \%)$. These results convey that people can use their knowledge of the stereotypical clock face numbering to facilitate response selection, and to a lesser extent can use numerical order when it differs from that of a stereotypical clock face.

Although the pattern of mapping effects for this symbolic stimulus set in Fitts and Deininger's (1954) study was qualitatively similar to that for the spatial stimulus set, the effect sizes were smaller: random-corresponding $=210 \mathrm{~ms}$ and $5.0 \%$ for the symbolic set versus $794 \mathrm{~ms}$ and $13.2 \%$ for the spatial set. The IT was longer and error rate higher for the most 
compatible symbolic mapping than for the most compatible spatial mapping, whereas they were shorter and lower for the random mapping. This size difference indicates that the stereotypical relation to the circular response array is stronger, or dimensional overlap higher (Kornblum, Hasbroucq, \& Osman, 1990), for the circular spatial stimulus array than for the symbolic stimulus set.

In Fitts and Deininger's (1954) study, participants performed two sessions of 64 trials each on different days, with only a single display and mapping. There was also a third display - a linear array of lights mapped compatibly (left-toright stimulus locations to clockwise-from-top response locations), incompatibly (right-to-left stimulus mapping), and randomly. Analysis showed a significant overall reduction of approximately $60 \mathrm{~ms}$ in IT from Session 1 to Session 2 . Even though the conditions with longer IT seemed to benefit a bit more from practice than those with shorter IT (see Deininger \& Fitts, 1955, Fig. 13), the interaction was nonsignificant, and the rank ordering of conditions was the same in both sessions.

Most studies of stimulus-response compatibility and related effects have used key-press responses in choice-reaction tasks with two or more alternatives (Proctor \& Vu, 2006). Before these studies were ever conducted, Fitts and colleagues had established that the compatibility effects were based in spatial representations of the response alternatives. Consequently, most accounts of the effects attribute them to spatial coding (e.g., Umiltá \& Nicoletti, 1990). However, use of key presses with the left and right hands has led some researchers to attribute similar effects to effector-selection processes. An example is that of object-based compatibility effects, for which responses are faster when the irrelevant left or right location of the handle of a graspable object is congruent with the left or right key press designated by the relevant stimulus dimension. Tucker and Ellis (1998) first reported this compatibility effect and attributed it to a grasping affordance activated for the corresponding hand, a view that continues to have advocates (e.g., Pappas, 2014) despite considerable research (e.g., Bub, Masson, \& Kumar, 2018; Proctor \& Miles, 2014) showing that the results are due to what Masson (2018) called "insidious spatial correspondence effects" (p. 222). Much confusion and needless theorizing about automatic activation of grasping actions independent of task goals could have been avoided if researchers had heeded the message from Fitts and colleagues' initial studies that stimulus-response compatibility effects are primarily spatial.

\section{Hick's law}

Perhaps even more widely known than stimulus-response compatibility effects is Hick's law (Hick, 1952), also identified as the Hick-Hyman law (Hyman, 1953). For equally likely alternatives, the law is usually characterized as
Reaction Time $=t+b \log _{2} N_{A}$,

where $N_{A}$ is the number of stimulus-response alternatives, $b$ is the slope, and $t$ is a constant for other factors influencing reaction time (see Proctor \& Schneider, 2018, for a review). Hick reported results of an experiment in which participants placed all 10 fingers on response keys and pressed the key corresponding to the location of a light that came on from among a "somewhat irregular circle" of 10 lights. In different trial blocks, the number of possible stimuli ranged from two to 10. The slope of the resulting function indicated that reaction time increased by $115 \mathrm{~ms}$ for each doubling of possible alternatives. Noting this result, Fitts (1964) reported an unpublished study by Fitts and Peterson of set size in which participants were to move a single finger from a starting position and touch whichever one of a set of lights came on. In this case, IT increased by only $17 \mathrm{~ms}$ for each doubling of the number of alternatives. This between-study comparison suggests that the major process contributing to the slope of the Hick's law function is not spatial response selection but effector selection.

Wright and colleagues have obtained more controlled evidence that selection of unimanual responses is not influenced much by the number of possible alternatives when the response is signaled by the target location, but the influence is substantial when the response is signaled by an associated symbol. Specifically, Wright, Marino, Belovsky, and Chubb (2007) found that, when signaled spatially, increasing the number of alternatives added little or no increase to IT. Prior to each trial, Wright et al. (2007) designated two or six locations among eight candidate ones, arranged in a semicircular array, as possible targets. The potential target locations were designated with unfilled circles, and when one of the circles was lit (i.e., filled in), the participant was to move a stylus from a start position at the bottom of the display monitor to the target circle location. The IT difference between the twoalternative and six-alternative displays was negligible, implying high stimulus-response compatibility.

Wright, Marino, Chubb, and Rose (2011) replicated this result and found similar results when the potential target locations were designated by filling in all of the outline circles except the target circle, to which the participant was to move the cursor. In a second experiment, the response location was designated by a $2-\mathrm{cm}$ line for which the starting end was at fixation in the center of the display screen and the orientation aligned with one of the potential target circles. This experiment also showed little influence of the number of alternatives on IT. Together, these experiments provide evidence that automatic orientation of attention to a peripheral stimulus that changes is not the main factor resulting in the negligible influence of number of alternatives. 
A study by Wright et al. (2019) used logic similar to that of Fitts and Deininger (1954), but with the semicircular eightlocation target array. They assigned letters (or symbols) to the target locations corresponding to those on which the left and right hands would be placed when typing: $\mathrm{A}, \mathrm{S}, \mathrm{D}$, and $\mathrm{F}$ to the left hand and J, K, L, and ; to the right hand, going in order from the leftmost to rightmost target locations. Half of the participants were expert typists, and half were novices, but all learned the associations between the symbols and locations prior to responding. The display of possible target locations was not labeled during the tasks that were performed. Participants were tested using the stylus-movement task of Wright et al.'s (2007, 2011) studies, responding in one condition to filling in of the target location and in another condition to a centered letter designating the target location. They also were tested with press responses of the corresponding keys on the keyboard, using the four fingers from each hand excluding the thumb, also responding to filling in of one circle to designate the target response or to a centered letter.

The results showed increases in reaction time consistent with Hick's law for conditions with key-press responses. For key presses, the reaction time increased strongly from two to six alternatives: Averaged across the spatial and symbolic stimuli, the increase was estimated to be $91 \mathrm{~ms}$ for each doubling of the number of alternatives for novices and $51 \mathrm{~ms}$ for expert typists. Similar, though smaller, estimates in IT were obtained when stylus-movement responses were signaled by a letter stimulus (50 ms for novice typists and $34 \mathrm{~ms}$ for experts), but as in Wright et al.'s $(2007,2011)$ prior studies, there was little influence of number of alternatives when those responses were signaled spatially. Across both novices and experts, the slope of the IT function for each doubling of spatial stimulus alternatives was $6 \mathrm{~ms}$ when trials with repetitions of the same stimulus and response from the immediately prior trial were removed. Moreover, in this study, as well as those of Wright et al. (2007, 2011), MT in the spatial task was only about $5 \mathrm{~ms}$ longer with six choices than with two choices, indicating a negligible effect of number of alternatives on execution of aimedmovement responses.

Similar findings have been obtained in the context of simulated human-computer interaction tasks of pressing a button on a touch screen or clicking on a button in response to a command (though not with circular arrays). Roy, Guiard, Bailly, Lecolinet, and Rioul (2015) had participants perform a task on a simulated screen of an iPhone displayed on an iPad tablet. Participants placed their right hand on a start button below the smartphone, which had a row of buttons aligned across its width near the bottom. When one button turned gray, the participant was to move and touch it as quickly as possible. The results showed no increase in IT from 5 to 15 buttons, and only 100 -ms increase from 15 to 40 buttons. This latter increase is likely a result of holding the horizontal bar width constant, which caused the width of the buttons to be inversely related to the number of buttons. As expected, based on Fitts's law (Fitts, 1954), the reduction in button widths as button number increased resulted in MT increasing from $450 \mathrm{~ms}$ in the 5-button condition to $900 \mathrm{~ms}$ in the 40-button condition. Liu, Rioul, BeaudouinLafon, and Guiard (2017) obtained comparable results when the task required moving a cursor on a computer screen, with a mouse, to the target button in a row constructed like that in Roy et al.'s study, though the row was displayed at the top instead of the bottom of the screen. As summarized by Liu et al., "Task completion time is dominated by movement time" (p. 519).

Wright et al. (2019) concluded from their results, "The data are well fit by a model built on the assumption that effectorselection adds nothing to RT in the conditions for which there is a single, known effector, the touching responses, but provides a substantial component of the uncertainty effect in the conditions with key-press responses, in which each possible response involves a different finger" (p. 562). Why is this point crucial? Kieras (2009) summed it up in a proceedings paper, "Why EPIC Was Wrong About Motor Feature Programming." Meyer and Kieras (1997) developed the EPIC (executive-process/interactive-control) architecture for modeling performance of cognitive tasks to include a motorsystem component that required time for specification of movement features. This aspect of the model was used to model the Hick's law relation in choice-reaction tasks. Kieras (2009) stressed, "This original feature of EPIC was simply an incorrect overgeneralization" (p. 6), due to a focus on tasks for which the responses were key-presses and/or the stimulus-response relations were of low compatibility.

\section{Empirical summary}

The studies with unlabeled response locations indicate the following:

\section{Hick's law}

8. When all responses are made with a single effector and involve moving to a location that corresponds to the stimulus location, response selection and execution occur rapidly and effortlessly for up to six alternatives.

9. For situations in which each response is mapped to a different effector, the time for response selection increases substantially as the number of alternatives enlarges.

10. Points 8 and 9 together imply that effector selection is the primary factor influencing the Hick's law relation when the stimulus-response mapping is spatially compatible.

\section{Stimulus-response compatibility}

11. When the mapping of stimulus locations is to noncorresponding response locations and does not 
follow a simple rule, response selection is slowed noticeably, even with a single responding effector.

12. When the mapping follows a simple rule (e.g., mirror reversal), the cost of responding to noncorresponding locations is greatly reduced.

13. The IT benefit for the compatible mapping is maintained across many sessions of practice, indicating that selection of a noncorresponding response (without effector selection) still requires additional time.

14. In the context of a secondary mental arithmetic task, the advantage for compatible spatial mapping apparently shifts from IT to error rate, suggesting that the benefit in response selection is maintained, even though initiation of the movement is delayed.

15. A mapping of numeric stimuli corresponding to the placements of the response locations on a clock face shows a response-selection benefit over an incompatible mapping, but the difference is smaller than for spatial location stimuli.

16. For a compatible mapping of letters to their keys on a keyboard row, reaction time increases for both expert and novice typists as the number of alternatives increases, even with stylus-moving responses, but experts familiar with assignments of letters to keys and fingers show roughly half the effect that novices do.

17. An implication of points 15 and 16 is that when symbolic stimuli are mapped to responses, there is a responseselection cost but still some benefit for maintaining known relationships.

\section{Visual selection and response selection in circular arrays labeled with digits}

In the studies of Fitts and Wright, the response locations were unlabeled and the unimanual responses were of a stylus or finger. This basic paradigm can be elaborated to incorporate other forms of compatibility by (a) using a computer mouse to control the movements in two dimensions of a cursor on the display screen and (b) labeling the response locations with numerals. We begin by noting that a typical mapping of mouse movement to cursor movement yields results similar to the stylus and finger movements. Dassonville, Lewis, Foster, and Ashe (1999) used a circular array of eight locations on a display, for which two, four, or eight of the locations were possible in a particular trial block. When the cursormovement response was to be made directly to the target location, IT was unaffected by whether two, four, or eight locations were possible. However, IT showed results similar to a standard Hick's law function when the response was to a location rotated $90^{\circ}$ counterclockwise from the stimulus location or when the stimuli were centered letters indicating compass directions (N, S, E, W). Thus, the limb needs to be moving compatibly to the target location unless the targetcursor mapping is spatially compatible. The labels provide an additional dimension for the responses that allow the response-label to response-location mapping to be varied.

The studies described in the rest of this section used 10choice circular stimulus arrays similar to the eight-item stimulus arrays of Fitts and Deininger (1954) and Dassonville et al. (1999), and the 12-item stimulus arrays of C. W. Eriksen and Hoffman (1973). The main difference is that the 10 locations were numbered 0 to 9 in order, analogous to clock faces. The relation between the mouse and cursor was always the typical direct positioning, which let the influence of the label mappings on IT and MT to be isolated. Thus, the tasks allowed matching a target stimulus digit to the corresponding label digit (visual search) to determine the signaled response location, with the relation of that location to the cursor-movement response always being spatially compatible.

\section{Mapping of digit labels to positions}

Bourne, Healy, Pauli, Parker, and Birbaumer (2005) conducted a study in which each target location was designated by a circle, with the top or bottom circle positions containing zero (for upright and upside-down displays, respectively), and the other circle locations containing digits in clockwise or counterclockwise order relative to the zero position (see Fig. 2). Thus, there were four different displays in their Experiment 1, each used by eight different participants. A computer mouse directly controlled movement of a cursor in a normal manner, and the task was to move the cursor from a centered start position (marked by an $\mathrm{X}$ ) to the position on the clock-face circumference designated by a digit that appeared immediately above the start position.

In this study, then, response-effect compatibility was not a factor because the cursor-effect motion corresponded to the mouse movement in all conditions. There was one dimension for the relevant stimulus (the centered digit's numeric value), but two relevant dimensions (clockwise/counterclockwise, upright/upside-down) for the target locations of the aimedmovement actions (the digit, which designated the location to which the cursor was to be moved). The clock-face displays differed in three ways from the standard upright clockwise display: left-right flip (labeled Upright counterclockwise in Fig. 2); up-down flip (Upside-down counterclockwise); $180^{\circ}$ rotation (Upside-down clockwise). Participants performed 500 trials with one clock face, and after a 5-min break, another 500 trials with the same clock face or one of the other three clock faces. Instructions emphasized responding as fast as possible, but stressed finding the target location prior to initiating movement of the cursor to that location.

Results showed that IT, MT, and total time (IT + MT) did not vary across the four clock-face arrays. This result is 


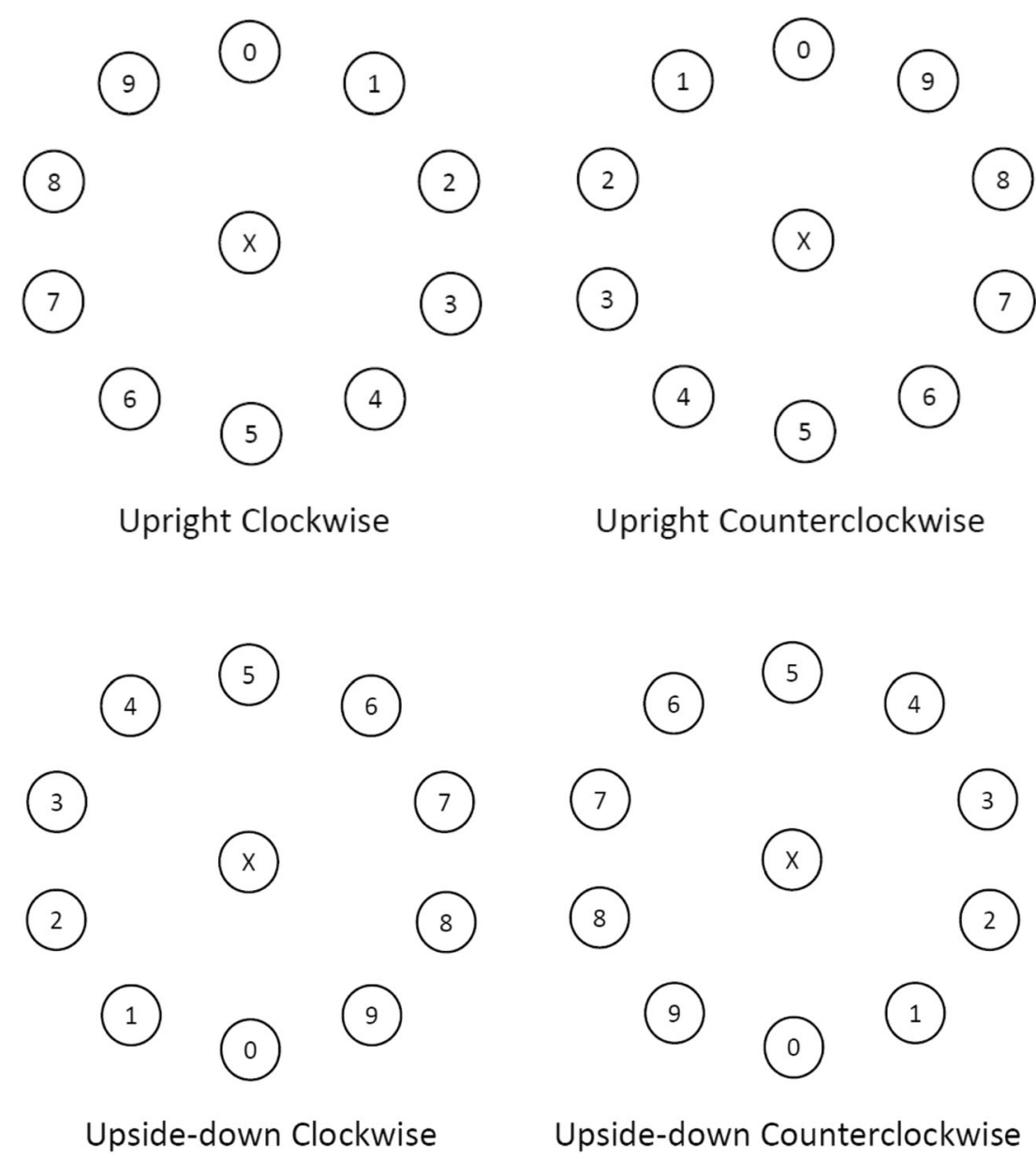

Fig. 2 Four different clock-face displays used in Bourne et al.'s (2005) Experiment 1

different from what Fitts and Deininger (1954) found for both spatial and symbolic stimulus sets, for which direct mapping yielded faster and more accurate responses than mirrorreversed mapping. The critical difference is that in Fitts and Deininger's study the response locations were not labeled, whereas in Bourne et al.'s study they were. Consequently, Fitts and Deininger's participants had to identify the signaled response location from a spatial-location stimulus (spatial stimulus set) or from an association of numeric stimuli with locations (symbolic stimulus set). In other words, spatial stimulus-response compatibility was higher with the direct mapping than with the mirror-reversed mapping. With the latter mapping, the response location signaled by a stimulus was different from the stereotypical stimulus location for all except the top (12:00) and bottom (6:00) positions.

With the labeled locations in Bourne et al.'s (2005) experiment, spatial compatibility is no longer a factor, but visual search is. The response location is identified by locating the target digit, after which the spatially compatible response is executed for all four display types. The absence of difference in performance across the display types shows that there is nothing inherently difficult about responding with alternative displays, as long as the location to which movement is to be made is not designated by a spatial stimulus or one with a stereotypical spatial relation, and the response/action is spatially compatible in all cases. Another way to describe this finding is that the stereotypical mapping of numbers to locations for the upright clockwise clock face does not provide any benefit in determining the numbered location to which to move when the display includes number labels. This lack of benefit is because participants can adopt a visual-search strategy of matching the centered digit to the corresponding digit at a response location based on the specific display configuration to which they are exposed. Because the digits' relations to the response locations were always consecutively ordered in Bourne et al.'s study, a search strategy could be adopted based on the display configuration without needing to retrieve number-location associations from memory.

Target digit also had a significant effect, with the effect larger on IT than on MT. The shortest times were for the digits 0 and 1 , and the longest for 6 . This result provides a clue to the search strategy employed. It suggests that 0 (which was at the top or bottom) served as an anchor, with search initiated from there toward the high end of the scale for most numbers and from the high to the low end for the largest numbers. 
Both IT and MT decreased in the practice session, with the primary reduction occurring over the first 60 trials. IT but not MT continued to show some reduction in the test session, suggesting that small improvements in response selection were still occurring. While the four clock faces yielded similar performance, a change in the specific clock face from the practice session to the test session produced a transfer cost compared with continuing with the same display. The cost was less for a left-right flip or $180^{\circ}$ rotation, each of which involves a change along only one dimension (clockwise/counterclockwise and upright/upside-down, respectively), than for an up-down flip, which involves change on two dimensions.

Thus, although practice was beneficial overall, the procedures acquired in the practice session were somewhat specific to the layout used: The more distinct the new interface was from the one with which the participants had practiced, the greater the cost of the change. Bourne et al. (2005) interpreted this result in terms of an expectations framework proposed by Fitts and Deininger (1954). According to this account, performance should be (a) best in the retention/ transfer session on the same clock face used in the practice session, (b) worst when expectations on both dimensions (clockwise/counterclockwise or upright/upsidedown) are violated, and (c) intermediate when only one of the expectations is violated. A possible basis for this pattern of results is that participants associate digits with responses to specific positions during the practice session, and these associations produce incorrect response tendencies in the transfer session.

Experiment 2 of Bourne et al. (2005) was similar to Experiment 1, but used only the upright clockwise and upside-down clockwise displays. The major difference was that during training participants were provided total response time feedback, either trial-by-trial or as the mean for each block of 20 trials immediately after the block's completion. No response-time feedback was provided in the test session. For the most part, the results were similar to those of Experiment 1. Again, there was a substantial cost in response time for the test session when the display array orientation was switched compared with when it was not. With regard to feedback, in the training session, IT but not MT was shorter in the trial-by-trial feedback condition than in the periodic feedback condition, suggesting that the immediate feedback motivated participants to search faster. Feedback during training did not affect test performance for either measure.

\section{Influence of arrow pointing to response location}

Pauli, Braun, Wiech, Birbaumer, and Bourne (2005) used the same clock-face method as Bourne et al. (2005), but in different trial blocks varied whether the centered target digit $0-9$ was presented alone or accompanied by an arrow pointing to the target location. The premise was that the arrow eliminated the need to identify the targeted location on the clock face because movement could be made in the direction indicated by the arrow. The arrow began at the center start circle and reached two-thirds of the way to the target on the clock face, and thus pinpointed the response location relatively directly. This method is similar to that of C. W. Eriksen and Hoffman (1972a, 1973), who used a black bar that extended outside the radius of a circular stimulus array to designate the target letter to report. An initial pilot experiment conducted by Pauli et al. used only the normal clock face, with 0 at the top and the other locations numbered in order clockwise. IT was approximately $200 \mathrm{~ms}$ shorter in the arrow condition compared with the number condition, but MT was not affected significantly. This result is in agreement with the premise that the arrow eliminated the need to search and identify the location indicated by the digit.

In the main experiment, each participant performed in two sessions. One session used the normal, ordered clock face, as in the pilot experiment, whereas the other used altered clock faces for which the locations of the digits varied randomly from trial to trial, with the order of the two sessions counterbalanced across participants. As expected, for the digit alone target condition, IT was much longer for the session in which the assignment of digits to locations varied randomly than in the session for which the digit assignment was constant and ordered (allowing a systematic search strategy to be employed). In contrast, for the arrow condition, there was no increase in IT for the randomized clock face, again in agreement with the presumption that matching of the target digit to a location was not needed. In contrast, MT showed no significant influence of the arrow manipulation or the clock-face numbering manipulation. So, once the target location was identified, MT did not depend on the time it took to make that identification, suggesting that there is a separate motor execution stage.

Note that the results obtained by Pauli et al. (2005) and Bourne et al. (2005) are in agreement with Kieras's (2009) conclusion, described earlier, regarding the EPIC architecture:

While successful in modeling many high-speed tasks involving choice reaction times with keypress responses, serious difficulties appeared in modeling high-speed $v i$ sual-search tasks involving saccades [not reviewed here] and mouse movements. A reappraisal of the basis for EPIC's assumptions requires a critical change: visually aimed manual and ocular movements require no feature preparation time. (p. 1, emphasis added)

Thus, although search for an alphanumeric target to identify the designated location takes time, little or no additional time is required to select a spatially compatible aimedmovement response.

\section{Empirical summary}

The studies with labeled clock faces yield the following conclusions: 
18. When response locations are labeled with ordered digits to which a target digit can be matched, it does not matter whether the numbering starts at the top or bottom, or proceeds in a clockwise or counterclockwise order.

19. Participants engage in an ordered search of the display, beginning at the zero location. This search does not rely on prior experience outside of the lab with a clock face, because the origin and direction of numbering does not matter.

20. Retention of a practiced mapping of ordered location labels to positions is good, but this learning does not transfer completely to other mappings, with the cost being larger when the switch in the later session involves two dimensions rather than one.

21. The results of point 20 imply that the search strategy acquired by participants from practice at the task is from the specific, practiced location labels in different directions for low and high numbers.

22. IT is considerably longer when the mapping of digits to response locations varies randomly from trial to trial and appears simultaneously with the target stimulus, but a line from the center that is aligned with the response location eliminates the cost of the random mapping. However, MT was not influenced by this manipulation, indicating that the effect lies in identification of the signaled response location.

23. The result in point 22 suggests that the line acts much like a corresponding spatial stimulus and eliminates the need for response selection (or identification).

\section{Response-effect compatibility for mouse and cursor movements}

With a mouse or other continuous control device, the mapping of the control movements to the cursor can be altered, as in the studies reviewed in this section. Although we focus on tasks that used numerical displays to identify target locations, we first describe how the response-effect mapping influences performance of an aimed-movement task when the target locations are not labeled. Cunningham (1989) had participants control a cursor with a stylus that they moved on a horizontal digitizing tablet. After centering the cursor, a target stimulus appeared at one of eight locations in a circular array, and the cursor was to be moved rapidly to that location. The styluscursor mapping was direct in some trial blocks, rotated by $45^{\circ}$, $90^{\circ}, 135^{\circ}$, or $180^{\circ}$ on the digitizer tablet in others, and reflected about the horizontal or vertical axis in still others. For the rotated conditions, MT was longest (and root mean square error [RMSE] of the trajectory greatest) for the $90^{\circ}$ and $135^{\circ}$ rotations, with IT showing a similar but nonsignificant pattern. Reflection about the vertical axis produced longer mean MT than reflection about the horizontal axis, but for both, RMSE (and presumably also MT) increased mainly for the locations on the axis of reflection (Cunningham \& Pavel, 1991). Cunningham (1989) proposed that the results were best fit by an account in which participants mentally align a bidirectional axis with the rotation, with more distant axes (near $90^{\circ}$ ) being less accessible. They then apply a direction of travel decision to that axis to generate the limb movement.

The remaining studies included in this section used a similar task to that of Bourne et al. (2005) to examine effects of training and transfer when the mouse-cursor relation rather than the numeric array relation was varied. Thus, these experiments assessed the influence of response-effect compatibility (Kunde, 2001) in a visual-search context. Most research on response-effect compatibility has been conducted from the standpoint of ideomotor theory (Shin, Proctor, \& Capaldi, 2010), according to which actions are represented in terms of their anticipated sensory consequences. Most of the research on response-effect compatibility has focused on its effects on response selection and initiation, although there is interest in whether it also affects response production (Kunde, Koch, \& Hoffmann, 2004). In most studies the effect is a discrete event (loud or quiet tone in Kunde et al.'s, 2004, study) that occurs after a response, so that any effect is restricted to anticipation of the event. However, the cursorpositioning response with a mouse is dynamic, which enables investigation of the influence of response-effect compatibility on the ongoing execution of the motor response.

\section{Variation in mouse-cursor mappings}

Healy, Wohldmann, Sutton, and Bourne (2006) conducted a study in which, after positioning the cursor on a center location (marked by $\mathrm{X}$ in Fig. 3), a target digit was presented designating the location in a circular display to which the cursor was to be moved. For all conditions, the display was the standard, upright clockwise clock face with only eight,

8

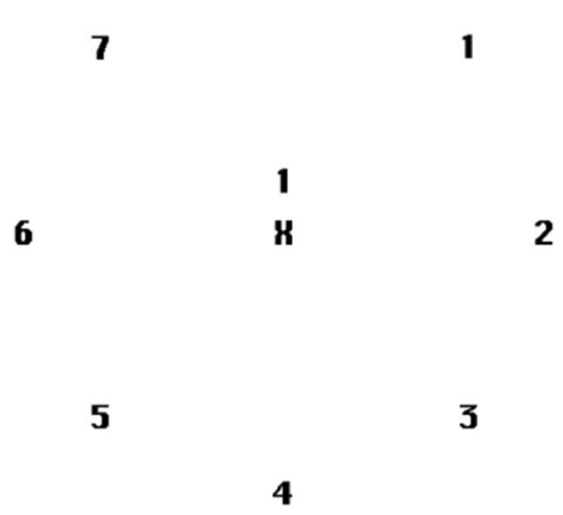

Fig. 3 Clock face display with the example target 1 used in Healy et al. (2006) 
rather than 10, digits (see Fig. 3). Thus, identification and selection of the signaled response location did not differ across conditions. Responses were made with a computer mouse that (a) directly controlled the mouse position in a normal manner or (b) was reprogrammed to produce reversed cursor movement on the horizontal dimension, vertical dimension, or both. Participants performed five blocks of 80 trials in each of two sessions, which were separated by a week.

Equal numbers of participants performed with each of the four mouse-cursor mappings in the practice session, and within each group, a quarter of the participants performed with each of the mouse-cursor mappings in the test session. The reported analyses focused on MT, although IT, described in an earlier draft of Healy et al.'s (2006) article, showed similar results. This relation is not surprising, because the responseeffect compatibility is a factor for the initial movement decision and throughout the movement to the target location.

In the training session, IT and MT were less for the normal mouse-cursor mapping than for the three altered mappings, which did not differ significantly. Thus, a benefit was evident for maintaining response-effect compatibility. Performance on both measures improved with practice, but more for the three altered mapping conditions than for the normal mapping. However, in the last of the five trial blocks of the training session, IT and particularly MT were still shorter with the normal mouse-cursor mapping than with the altered mappings. All these results are similar to those obtained with stimulus-response compatibility (Fitts \& Seeger, 1953).

Retention of performance was good for participants who continued with the same mouse-cursor mapping in the test session, regardless of which of the four mappings they used. All mapping conditions showed shorter IT and MT in the first block of the test session than in the last block of the training session, with MT for the three altered mapping conditions showing the greatest reduction. Participants who transferred from the horizontal or vertical reversal condition in practice to the opposite reversal condition in the test session showed negative transfer on both IT and MT. This result is consistent with Cunningham's (1989) conclusion that when performing with an altered mouse-cursor mapping, participants adopt a bidirectional reference axis that is specific to the reflected dimension. Likewise, negative transfer was evident in both measures when participants practiced with the combined horizontal and vertical reversal condition and performed with reversal on only one of the dimensions in the test session. This specificity of transfer implies that mouse-cursor mapping in the combined condition is not represented as separate reversals on horizontal and vertical dimensions.

However, training with a single dimension (horizontal or vertical reversal) improved performance in the combined horizontal and vertical test condition. Therefore, some positive transfer of part-to-whole response-effect mapping occurred, even though transfer from whole to part did not. This latter part-whole transfer may arise from some basic properties of the practiced reversal (e.g., horizontal) being relevant to the combined reversal mapping (e.g., to move the cursor right, make a leftward movement of the mouse).

In both sessions, with the horizontal reversal MTs were shortest for the digits 2 and 6 , located on the horizontal axis, whereas with the vertical reversal MTs were shortest for the digits 4 and 8 , located on the vertical axis. The conditions with the normal mouse-cursor relation and the combined horizontal and vertical reversal showed no substantial difference in MT among the target digits. The lack of advantage for the horizontally and vertically aligned digits in the combined condition also implies that spatial representation of the mouse movement and cursor action is not an independent combination of those for the horizontal and vertical dimensions.

A notable finding in the first 80-trial block of Experiment 1 was that for the horizontal reversal and vertical reversal conditions, MT was less to the digits along the axis of reversal than to the digits on the axis that was not reversed. Moreover, MT for digits on diagonals was less for the combined condition than for the horizontal or vertical condition, consistent with the other results indicating that representation of mouse-cursor movement in the combined condition is not the sum of the reversals along the two respective dimensions. In the first block of the test session, transfer from the horizontal reversal to vertical reversal, or vice versa, resulted in faster responding on the current reversed dimension than on the one that was reversed during training. One way to think about this result is that the nonreversed dimension in practice intruded less on performance in the transfer session than did the dimension that was reversed in practice.

Experiment 2 examined whether explicit instructions to use a strategy of moving toward the mirror opposite stimulus would lead to shorter MTs when the mouse-cursor mapping was horizontally reversed. All participants received that mapping condition and were told that the horizontal axis of the mouse was reversed. Unlike in Experiment 1, half of the participants were given explicit instructions about using a reflection strategy, whereas half were told not to use any strategy. Results showed a statistically significant difference in the pattern of MTs across target digits between the two strategy conditions, but the difference was small. The pattern of results for MT was similar in both conditions, showing MT advantages for the targets on the horizontal axis of similar magnitude. These results suggest that use of an explicit reversal strategy played at most a minimal role when executing responses with the altered mouse-cursor mappings. The results for IT also showed little influence of the instruction condition, but in both conditions IT was an inverted U-shaped function of digit magnitude, consistent with a strategy to locate the signaled target by serially searching the clock-face array, starting at the highest or lowest digit. 
In Experiment 3, each session contained 12 blocks of 32 trials. Participants were assigned to one of five training conditions: vertical reversal only; horizontal reversal only; vertical + combined reversal; horizontal + vertical + combined reversal; and normal + vertical + combined. For the vertical + combined training condition, there were six blocks of each type, in alternating order. For the training conditions with three reversal conditions, there were four blocks of each type, again alternating in order. Of concern was the second, transfer session, in which all participants performed with the horizontal reversal. For both IT and MT, the strongest advantage in that session was found for training in the horizontal-only condition, which is to be expected since it was a retention test. The next strongest advantage was for training with some blocks of horizontal reversal (horizontal + vertical + combined). Performance was worse for the other training arrangements, which did not differ significantly from each other, even though they differed in having one, two, or three reversal conditions. Thus, specific practice with the horizontal reversal, even when embedded in blocks with other mappings, was essential for positive transfer to the horizontal reversal condition in the second session.

Healy, Wohldmann, and Bourne (2011) reported results of an experiment that was similar to that of Healy et al. (2006), with the major change being that the test session was administered $5 \mathrm{~min}$ after the training session ended, rather than a week later. The main reason for this change was to allow assessment of transfer from the reversed mouse conditions to the normal mouse condition that would not be contaminated by participants' using a normal mouse in their intervening daily activities. Their Fig. 5 shows that in the training session, again, the shortest ITs were for the two extremes of the number order (1 and 8), with longest IT being for the digit 4 , with only slight interaction with mouse-cursor mapping condition. This result implies that a similar search strategy is employed regardless of the level of response-effect compatibility.

Of most interest, however, was transfer from those participants who practiced with mouse-cursor reversals on the horizontal dimension, vertical dimension, or both in the training session and performed with the normal mouse-cursor relation in the test session. For participants trained in any of the reversed mouse conditions, IT, and particularly MT, were longer with the normal mouse than with the reversed mouse in the test session, despite the fact that during training responses were considerably faster in the normal mouse condition than in the reversed mouse conditions. Thus, even an overlearned spatial relation between mouse and cursor movement can be overridden with one session of practice with an atypical mapping. However, this negative impact on performance with the normal mouse in the test session dissipated quickly, being evident primarily in the first test block of 16 trials.
The studies by Healy et al. $(2006,2011)$ are consistent with the view that the variables of digit label (1-8), which should affect the time to search and identify the target location, and response-effect compatibility, which should affect the time to initiate and prepare the required response, have their influences on distinct stages of information processing. Of most relevance for understanding action control, the results illustrate that response-effect compatibility has a large, ongoing influence on execution of aimed movement responses, in addition to an effect on the initial response selection. Koch, Keller, and Prinz (2004) emphasized that when considered as sensory feedback resulting from a response, response effects have been viewed as playing a significant role in motor control for many years (e.g., Adams, 1968). Feedback is regarded as essential for both acquisition of motor skill and online control of all but the fastest movements. Therefore, it seems likely that the influence of response-effect compatibly on MT is on the selection of submovements to correct errors in the movement trajectories to land accurately on the target location (Fitts, 1954; Meyer, Abrams, Kornblum, Wright, \& Smith, 1988). An implication is that these corrections may themselves be response-selection decisions that can be made faster and more accurately when the mouse-cursor mapping is response-effect compatible than when it is not.

\section{Empirical summary}

The studies on response-effect compatibility with variation in mouse-cursor mappings yield the following conclusions:

24. Incompatibility between mouse movements and cursor motions lengthens IT and MT, which is a responseeffect compatibility effect.

25. Retention across a week was good for all practiced mouse-cursor mappings.

26. Transfer from combined horizontal and vertical reversal to conditions with reversal on one dimension was negative, whereas transfer from either individual dimension to the combined horizontal and vertical test condition yielded positive transfer.

27. The outcomes in point 26 indicate that positive wholepart transfer did not occur, whereas positive part-whole transfer did. Reversal on both dimensions was evidently not processed as an additive combination of separate horizontal and vertical reversals.

28. MTs for horizontal reversal were shortest for the digits located on the horizontal axis, but MTs for vertical reversal were shortest for the digits located on the vertical axis, suggesting mental alignment with the axis of rotation.

29. For positive transfer to a horizontal reversed mapping from practice with various mappings to occur, 
participants must have some experience with the horizontal mapping during the training session.

30. When performing with a horizontally reversed mousecursor mapping, instructions to adopt an explicit reflection strategy have little influence on performance.

31. Practice with a reversed mapping on either the vertical or horizontal dimensions, or both, creates negative transfer to a condition with the normal mapping between mouse and cursor movement, but this negative transfer is short lived.

\section{Variation in trained targets}

Wohldmann, Healy, and Bourne (2008) reported two experiments similar to those of Healy et al. (2006, 2011), with a 5min interval between the training and test sessions. All participants practiced 12 blocks with 16 trials each for the training session and then a second set of 12 blocks for the transfer session. The horizontal reversal condition was used for the mouse-cursor relation for both sessions, which means that the relation of the mouse movement and the cursor action effect was constant across all conditions. Four groups received distinct subsets of four targets during the training session: In Experiment 1, participants practiced moving to targets on the horizontal (2 and 6) or vertical (4 and 8 ) dimension, and on one of two diagonal dimensions ( 1 and 5 , or 3 and 7 ). Trials with all eight target stimuli and locations occurred in the test session. Thus, for all groups two of the targets (those on a diagonal) required movement of the mouse on both reversed and nonreversed dimensions. Movements for the other two targets were along only the reversed (horizontal group) or nonreversed (vertical group) dimensions.

The reported analyses focused on the diagonal target locations in the test session, two of which had received practice in the training session and two of which had not. Results showed evidence of specific learning for both IT and MT and general learning for MT. The two groups of participants trained with the negative diagonal locations 3 and 7 showed only a negligible 9-ms longer IT to new targets than to old targets, whereas for the two groups trained with the positive diagonal locations 1 and 5, IT averaged $112 \mathrm{~ms}$ longer to new targets than to old targets. This asymmetry may reflect more time being spent planning motor movements on the negative diagonal due to biomechanical factors that make movement more difficult on the that diagonal than on the positive diagonal (Cunningham \& Pavel, 1991; Keele, 1968). Averaged across the two diagonals, the 60-ms advantage for old targets implies learning specificity.

Despite this difference in IT for the positive and negative diagonals, MT showed a main effect of target type, being more than $250 \mathrm{~ms}$ longer overall for new targets than old ones, with this difference much larger in the first trial block (approximately $600 \mathrm{~ms}$ ) than in the later blocks. This difference may be due to the aforementioned biomechanical differences for movements that are executed on the positive and negative diagonals. This effect of target type on MT also indicates considerable specificity of transfer, particularly initially, to the practiced relations. Comparisons of the first five blocks of trials for participants on the new diagonal targets in the test session to the first five blocks of training for participants who began with those same diagonal targets showed no evidence of positive transfer for IT, but significantly shorter MT in the test session. Thus, movement planning apparently was specific to the trained targets, whereas movement execution had a generalizable component as well.

Experiment 2 was similar to Experiment 1, but with participants being trained with only two targets on a single axis (vertical, horizontal, negative diagonal, positive diagonal). Thus, in this case, experience in the practice session was with either the nonreversed dimension, the reversed dimension, or movements requiring a combination of reversed and nonreversed dimensions. Similar to Experiment 1, participants who trained on one of the diagonal axes showed shorter IT and MT to old than to new targets. Again, the difference in IT between old and new targets was larger for participants trained on the 1 and 5 diagonal than for those trained on the 3 and 7 diagonal. MT also showed some evidence of positive transfer compared with the training session, whereas IT again did not.

Because the test sessions in Experiments 1 and 2 were identical and the participants came from the same population in the two experiments, comparison across experiments of participants who performed with diagonal stimuli in the practice session provides some indication as to whether it was better to practice with nondiagonal stimuli intermixed (Experiment 1) or not (Experiment 2). Comparisons for IT and MT as a function of experiment, old or new target diagonal, and trial block showed a significant interaction of experiment and old/new diagonal only for MT. Basically, the results showed little difference in MT between the experiments for responses to old targets, whereas MT to new targets benefited from the more variable practice provided in Experiment 1. Another way to characterize this result is that practice responding to a wider variety of targets allows the participant to learn the movement characteristics of the mouse and cursor better, producing positive transfer to the new diagonal.

Wohldmann and Healy (2010) again trained and tested participants to move a cursor from the start position to the target position with mouse-cursor reversal on only the horizontal axis in all cases. As in Wohldmann et al. (2008), participants performed 12 blocks of 16 trials in both training and test sessions. In training, all trials for condition D1 were targets on one of the two diagonals ( 1 and 5 , or 3 and 7), for condition D2 the targets were one digit from each diagonal and located on the same side of the display (1 and 3, or 5 and 
7), and for a control condition the targets were all eight digits. All eight targets were tested equally often in the test session.

For training, IT was longer in the control condition, for which there were eight alternatives $(M=896 \mathrm{~ms})$, than in the $\mathrm{D} 1(M=639 \mathrm{~ms})$ and $\mathrm{D} 2(M=695 \mathrm{~ms})$ conditions. The longer IT in the control condition is expected on the basis of Hick's law (Hick, 1952; Proctor \& Schneider, 2018), but the difference between the latter two conditions is not. The shorter IT when the two possible targets shared a common diagonal is likely because a left-right decision could be made as part of the selection process when the possible targets were on the same diagonal, but not when they were on different diagonals. IT decreased for all conditions as a function of practice, although somewhat less for the D1 condition than for the other two conditions, because IT was already substantially shorter for it in the first trial block. MT also decreased across trial blocks, but showed no main effect of, or interaction with, condition. Once the target was identified, the number and nature of the original possible targets mattered little.

Retention was evaluated by comparing performance on the last five blocks of training in the control condition to that on the first five blocks of testing in the D1 and D2 conditions for old targets. IT showed no significant difference between the three conditions, but the mean IT tended to be longer in the D1 $(M=937 \mathrm{~ms})$ and D2 $(M=876 \mathrm{~ms})$ conditions than in the control condition $(M=799 \mathrm{~ms})$. For MT, the difference was significant, with MT being shorter in the D1 $(M=1,011 \mathrm{~ms})$ and $\mathrm{D} 2$ conditions $(M=916 \mathrm{~ms})$ than during training in the control condition ( $M=1,196 \mathrm{~ms})$. Thus, whereas there was possibly a slight cost in IT of having performed with only a subset of targets in the training session, there was a benefit of practicing with a subset of targets for MT on the old targets.

Specificity of training was evaluated by comparing performance differences to old and new targets in the test session for the D1 and D2 conditions. IT was shorter for old $(M=782$ $\mathrm{ms})$ than new $(M=856 \mathrm{~ms})$, as also was the case for MT (old: $M=881 \mathrm{~ms}$; new: $M=1,125 \mathrm{~ms}$ ). Thus, both measures showed evidence of specificity of training. Near transfer to the new diagonal targets and far transfer to the horizontal and vertical target locations were evaluated in several ways, including assessments of partial and full transfer. Positive transfer was evident in MT (largely reflecting execution of movements), which was shorter in the D1 and D2 conditions to new targets during testing than to those same targets in the control condition during training, with full transfer evident for D2, but only partial transfer for D1. In contrast, IT (largely reflecting encoding and planning of movements) showed no indication of even partial transfer to the new targets. These results show that specificity and transfer can both be evident for training a given task - that is, they are not mutually exclusive. Furthermore, the presence of transfer depends on the particular processes being measured, suggesting in this case more transfer of the processes involved in executing movements than of those involved in encoding or planning movements.

\section{Empirical summary}

The studies on response-effect compatibility with variation in targets yield the following conclusions:

32. With a horizontal reversed mouse-cursor mapping, movement planning (IT) was specific to the targets on the trained diagonal and did not transfer to the new targets on the untrained diagonal, whereas movement execution (MT) also had a generalizable component that benefited responses on the untrained diagonal.

33. MT to new targets showed a larger benefit when practice was with the targets on one diagonal intermixed with targets on one of the dimensional axes than when only trials on that diagonal were practiced. This result implies that practice responding to a wider variety of targets allowed the participant to learn the general movement characteristics of the mouse and cursor better.

34. IT on old targets showed a slight cost of having performed with only a subset of targets in the training session, but MT to the old targets showed a benefit of performing with that subset of targets.

35. IT showed little or no transfer from practice with two diagonal stimuli to performance with new stimuli, whereas MT did show positive transfer. This result pattern implied that there is more transfer of the processes involved in executing movements than of those involved in preparing or planning the movements.

\section{Variation in focus of attention}

Considerable evidence has been gathered for the view that an external focus on action outcomes results in better learning and performance than an internal focus on the muscular movements involved (Sherwood, Lohse, \& Healy, 2020; Wulf \& Lewthwaite, 2016). Carpenter, Lohse, Healy, Bourne, and Clegg (2013) investigated this issue with the clock-face task. Participants performed under two reversal conditions, which could be either the same or different in two sessions. For one condition, the mouse and cursor directions were reversed only along the horizontal dimension, and for the other they were reversed on both horizontal and vertical dimensions. Half of the participants who received each reversal condition in the first session also performed with that reversal condition in the second session, a week later, whereas half switched to the alternative reversal condition. Within each Session 1 reversal condition, half of the participants received internal focus instructions to attend to the direction in which their hand controlling the mouse moved, and half received external focus 
instructions to attend to the direction in which the cursor on the screen moved.

Performance for all participants improved within each session and was better in Session 2 than in Session 1, showing benefits of training. Participants instructed to adopt an external focus showed greater reduction in both IT and MT across blocks in Session 1 than did those instructed to adopt an internal focus, and they responded with faster performance during the early trial blocks of Session 2. Thus, external focus led to quicker acquisition and better maintenance of the speeded responses over the week retention interval than did internal focus. Participants who performed the task with horizontal reversal in Session 1 performed faster overall in Session 2, regardless of whether they were tested with that same reversal condition again or with the condition for which there was both horizontal and vertical reversal. Participants who began with the horizontal + vertical reversal condition in Session 1 showed worse performance when they switched to the horizontal reversal condition in Session 2. That is, the transfer data showed positive part-whole transfer but negative whole-part transfer (as in Healy et al. 2006, 2011). An implication is that participants were not attending in training specifically to the horizontal reversal relation when it was combined with reversal on the vertical dimension.

The lack of whole-part transfer from reversal on both dimensions (horizontal and vertical) to conditions with reversal on only one dimension (horizontal or vertical) in Carpenter et al.'s (2013) and Healy et al.'s (2006, 2011) experiments has implications for modeling representations for cursor control. Murata (1999) modeled positioning of a mouse-controlled cursor from an initial lower left position on the display screen to a target box in the upper right corner with independent probability distributions on the horizontal and vertical dimensions. Although mouse positioning with a normal mousecursor mapping may be coded by performers along the two separate dimensions, the negative transfer from the condition with reversals on both dimensions to conditions with reversal on only one dimension in Healy et al.'s $(2006,2011)$ studies implies that independence is not generally the case.

That positive transfer did occur when the transfer was from part to whole is consistent with the specificity of training principle (Healy \& Bourne, 2012). According to this principle, practice will be beneficial to the extent that the procedures acquired in practice are applicable in the transfer task. In the case of reversed mappings, practicing with a horizontal reversal, for example, would be expected to transfer to the task with reversals on both dimensions. This positive transfer would be because, even with a top-bottom reversal added, leftward mouse movement will still produce rightward cursor movement. The finding in the study by Healy et al. (2006, Experiment 3) that positive transfer to the horizontalreversed mapping occurred only when at least some of the practice was with that mapping is also consistent with the specificity of practice principle.

\section{Empirical Summary}

The studies on response-effect compatibility with variation in focus of attention yield the following conclusions:

36. In the clock-face paradigm, both IT and MT improve more with practice and are maintained better over a 1week interval when participants are instructed to attend to the cursor (the response effect) rather than the mouse movement (the physically controlled manipulandum).

37. Consistent with the data of Healy et al. (2006, 2011), positive transfer occurred from practice with the horizontally reversed mapping to the mapping with reversal on both dimensions but not vice versa.

\section{Discussion}

The research on studies using circular displays and no effector selection has shown consistent and clear patterns of results. Phenomena that are evident in these studies therefore cannot be attributed to effector selection. This is a point that seemingly was appreciated by Eriksen and colleagues (e.g., C. W. Eriksen \& Hoffman, 1973) and Fitts and colleagues (e.g., Fitts \& Deininger, 1954), who were interested in studying visual selection and response selection, respectively, and not effector selection. Wright and colleagues (e.g., Wright et al., 2019) have highlighted this fact in recent years. When the singleeffector responses allow movements to a targeted response location, the circular-array paradigm allows for separation of IT and MT, which sometimes show different effects. When the responses control a cursor, effects of compatibility between responses and their effects on the cursor movements can be studied. Finally, by altering various aspects of the displays and mappings after practice, precisely what has been learned during the initial practice can be determined.

Extending back to Donders (1868/1969) and Sternberg (1969), a century later, researchers have proposed that performance of complex cognitive tasks can be decomposed into distinct stages of information processing that are relatively independent. The findings we have covered are comprehensible within such a framework that separates visual selection from initial response selection and selection of corrective actions when executing movements.

\section{Visual selection in circular arrays}

The experiments of Eriksen and colleagues, along with many others, indicate that the time to select and identify a target 
letter in a circular array increases as a function of the display size. More important, they show that visual attention can be directed to a specific location in a circular array, but 150-350 $\mathrm{ms}$ is required to do so if the location is cued in advance of the target. The limit of the focus of attention is about $1^{\circ}$ of visual angle, but it can be expanded to a larger area if needed. Their research establishes that flanking stimuli within the focus of attention produce interference with selection of the response to a designated target when they are assigned to an alternative response. Thus, the locus of the flanker interference effect must be in activation produced in the response-selection process, as reflected in Eriksen's emphasis on response competition (e.g., C. W. Eriksen \& St. James, 1986). Because the research from Eriksen's lab uses vocal naming and unimanual left-right lever-push responses, the results also establish that flanker interference occurs at a more central level of response selection than effector selection.

\section{Response selection with circular stimulus and response arrays}

The experiments of Wright and colleagues (e.g., Wright et al., 2019) show that for tasks in which stimulus location is the relevant information, and the response is movement of a single effector to a corresponding location, response selection and execution occur rapidly and effortlessly for up to six alternatives. This outcome is in contrast to tasks in which each response is mapped to a different effector, for which Hick's law applies and reaction time increases as a logarithmic function of the number of alternatives (Proctor \& Schneider, 2018). The implication is that selection of the location at which to respond is fast and effortless with a compatible spatial mapping. The separate process of effector selection required for key presses executed by distinct fingers is what creates difficulty as the number of alternative stimuli and responses increases.

As shown by Fitts and colleagues (e.g., Fitts \& Deininger, 1954), when stimulus locations are mapped arbitrarily to noncorresponding response locations, response selection is slowed noticeably, even with a single responding effector. This outcome is in agreement with many other findings implying that spatial compatibility effects are mainly a consequence of the overlap in spatial dimensions of the stimuli and responses (Proctor \& Vu, 2006). The benefit for the compatible mapping is reduced but still evident across many practice sessions, indicating that selection of an incompatible response requires additional time even when a participant is highly practiced. An implication is that "automatic activation" of the corresponding response is amply strong and that it continues to interfere with selection of the correct response location even when the alternative mapping is well practiced. That the Eriksen flanker effect and the stimulus-response compatibility effect both occur for tasks that require no effector selection suggests that the cost of both types of incompatibility has a similar basis in activation of a competing response in the response-selection process.

For a compatible mapping of numeric stimuli corresponding to the placements of the response locations on a clock face, a stereotypical, spatially compatible mapping to responses shows a benefit over a random mapping. This result indicates that people's knowledge of clock faces, possibly through use of imagery, provides a basis for facilitating response selection when the alternative locations also correspond to those on a clock face. Even when an ordered mapping of numbers to response locations is mirror opposite to that of a stereotypical clock face, the ordinal relation provides some benefit to response selection. For a compatible mapping of letters (or symbols) to their keys on a keyboard row, response time increases for both expert and novice typists as the number of alternatives increases, even with stylus-moving responses, but the experts show roughly half the effect size that the novices do (Wright et al., 2019). These findings imply that when symbolic stimuli are mapped to responses, there is a response-selection cost that cannot be attributed to effector selection. That is, activation of corresponding response locations still occurs, but to a lesser extent than for physical stimulus locations and responses.

\section{Visual selection and response selection for locations labeled with digits}

When visual search is combined with spatial compatibility, and responses are those of a cursor controlled by a computer mouse, as in the studies of Bourne et al. (2005) and Pauli et al. (2005), the findings implicate visual search for a designated target digit. Unlike when the response locations are unlabeled (Fitts \& Deininger, 1954), there is no benefit of maintaining a stereotypical clock face, with origin at the top and clockwise order, compared with another origin and counterclockwise order. However, participants still are able to take advantage of the ordering provided by the numbers: When response locations are labeled with ordered digits, participants engage in search of the display beginning at the zero location, regardless of whether the numbering starts at the top or bottom or is clockwise or counterclockwise. They proceed in this ordered search until a match for the target digit is found, which identifies the response location. This depiction is based on the finding that initiation time, but not movement time, was fastest for 0 and 1 and increased for numbers in either direction, yielding a bow-shaped pattern for which initiation time was longest for 6 (at the location opposite from 1). It is worth emphasizing that this visual-search process for labeled arrays circumvents the benefit of prior experience with a specific configuration that is evident when the array is not labeled.

As expected from studies of visual search, it takes considerably longer for participants to respond when the mapping of digits to response locations varies randomly from trial to trial 
and the labeling appears simultaneously with onset of the target stimulus. However, a line from the center that is aligned with the response location eliminates the cost of the random mapping. Because IT is influenced by this manipulation but MT is not, this effect also lies in identification of the signaled response location. That is, the line acts much like a corresponding spatial stimulus and eliminates the need for identification of the signaled response location.

With regard to practice and transfer, retention of the practiced mapping of ordered location labels to positions in a subsequent test session is good, but there is not complete transfer to other mappings. The cost of changing a mapping of digits to response locations is greater when the change involves two dimensions rather than one. These results indicate that there is a degree of specificity to learning the ordered search strategy. That is, for example, if a participant has performed many trials starting with 0 at the bottom and scanning upward left for a low digit and upward right for a high digit, the scanning strategy must be relearned when those relations are changed. This learning shows up in the test session as an effect on IT (time to locate the target digit). MT shows no change in the test session, because once the location has been determined, the movement is the same as in the practice session.

\section{Movement planning and execution as a function of response-effect compatibility}

The studies of Healy and colleagues with altered mousecursor mappings are the only ones for which the emphasis is on movement planning and execution. In all conditions, the arrays are labeled consistently with an order similar to a clock face, and the location to which the cursor is to be moved is signaled by a target number. In their experiments, when the mapping of mouse movements to cursor movements is varied, incompatibility between mouse movements and cursor motions lengthens IT and MT, which is defined as a responseeffect compatibility effect. This incompatibility affects the time to determine the initial movement, as in most studies of response-effect compatibility and, because it is present throughout the movement, the time to execute movement of the cursor to the target location. The influence on MT may be attributable to anticipation of effects of selection of corrective actions, much like on selection and planning of the initial response (Shin et al., 2010).

Retention across a week is good for all practiced mousecursor mappings, implying that the learning of even incompatible mouse-cursor relations is relatively durable. Transfer from combined horizontal and vertical reversal to conditions with reversal on one dimension is negative, whereas transfer from either individual dimension to the combined horizontal and vertical test condition is positive. Thus, positive wholepart transfer does not occur, whereas positive part-whole transfer does. This outcome implies that reversal on both dimensions is not processed as an additive combination of separate horizontal and vertical reversals. More generally, the negative whole-part transfer underscores the high degree of training specificity evident with these tasks, so that identical individual stimulus and response elements (e.g., Thorndike, 1906) might not be sufficient for transfer. Rather, successful training might require using the same full configurations of stimuli and responses during training and testing.

With a horizontal reversed mouse-cursor mapping, movement planning (IT) is specific to the targets on a trained diagonal and does not transfer to the new targets on the untrained diagonal, whereas movement execution (MT) has a generalizable component as well that benefits responses on the untrained diagonal. IT on old targets shows a slight cost of participants having performed with only a subset of targets in the training session, but MT to the old targets shows a benefit of performing with that subset of targets. IT shows little or no transfer from practice with two diagonal stimuli to performance with new stimuli, whereas MT does show positive transfer. This result pattern implies that there is more transfer of the processes involved in executing movements than of those involved in planning the movements. Studies that use a single measure of response time without separating IT and MT might provide an unclear or misleading picture of transfer and the processes underlying learning.

Finally, results obtained with manipulations of instructions for the horizontal reversed mouse-cursor mapping are informative. Explicit instructions to use a reflection strategy have minimal influence on performance compared with giving no such instructions. This result suggests that the learning that occurs when using an altered mouse-cursor mapping has little relation to explicit intentions of the participants. Instructions to focus on the cursor element yield better acquisition and retention of performance than do instructions to focus on the movements of the mouse. Explicit intent to execute a strategy does not seem to be beneficial, and an explicit focus of attention on the movements of the mouse is in fact harmful.

\section{Conclusion}

The reviewed studies highlight the value of systematically studying human performance in tasks for which effector selection is not a factor. The findings indicate that many, but not all, crucial perceptual, cognitive, and movement-related effects occur in these tasks. Visual search is a time-demanding process that increases with the number of potential target locations in an array. However, it can be largely bypassed by allowing people time to focus attention on a single cued location, and this attentional focus can be enlarged to encompass more than one location. Without a cue, people can use ordered search strategies to identify numerically labeled locations in an array, and the location of initiation and the direction of magnitude in the order do not matter much, as long as those 
relations are held constant. With unlabeled response locations, people can also use an ordered mapping of numbers to facilitate response selection, but in this case prior associations of the numbers with specific locations on a clock face facilitate performance beyond that attributable just to the ordered numerical relation.

The aforementioned processes of visual selection and response selection occur in tasks for which movements of the effector and response-effect feedback are held constant. Manipulations of mouse-cursor relations allow the movement-related processes to be studied, as do those that require effector selection on a keyboard. For the most part, the visual selection and response selection aspects of performance are amenable to explicit intentions and strategies, and are under cognitive control, whereas those involving movement do not seem to be. The influence of response-effect compatibility during movement execution likely involves cognitive decisions about corrective actions that are implemented by the motor system. These conclusions are in accord with those reached by Logan and Crump (2011) in their studies of transcription typing. Their model distinguishes an "outer loop" of cognitive control from an "inner loop" of motor control. Logan and Crump have provided extensive evidence that the outer loop sets the action goal (e.g., typing a specific word) and is affected by different variables than the inner loop, which controls the physical movements to accomplish the goal (pressing the appropriate keys). The outer loop provides input to the inner loop, but it does not have access to the state of the inner loop or how it accomplishes its goal. Effector selection, and motor control in a range of tasks seem to be the province of the inner loop, and not of the decisions and goals of the outer loop, although decisions about corrective actions likely are.

Author note Preparation of this report occurred while Alice Healy was a visiting scholar in the laboratory of Professor Michael Kahana at the University of Pennsylvania.

\section{References}

Adams, J. A. (1968). Response feedback and learning. Psychological Bulletin, 70, 486-504.

Bourne, L. E., Jr., Healy, A. F., Pauli, P., Parker, J. T., \& Birbaumer, N. (2005). The influence of stimulus array on training of a speeded response. American Journal of Psychology, 118, 385-411.

Bravo, M. J., \& Nakayama, K. (1992). The role of attention in different visual-search tasks. Perception \& Psychophysics, 51, 465-472.

Bub, D. N., Masson, M. E. J., \& Kumar, R. (2018). Time course of motor affordances evoked by pictured objects and words. Journal of Experimental Psychology: Human Perception and Performance, 44, 53-68.

Carpenter, S. K., Lohse, K. R., Healy, A. F., Bourne, L. E., Jr., \& Clegg, B. A. (2013). External focus of attention improves performance in a speeded aiming task. Journal of Applied Research in Memory and Cognition, 2, 14-19.
Cave, K. R., \& Chen, Z. (2016). Identifying visual targets amongst interfering distractors: Sorting out the roles of perceptual load, dilution, and attentional zoom. Attention, Perception, \& Psychophysics, 78, $1822-1838$.

Chen, J., \& Proctor, R. W. (2015). Influence of response-effect feedback on learning and performance of a complex key-pressing task: Morin and Grant (1955) revisited. American Journal of Psychology, 128 , 197-208.

Colegate, R. L., Hoffman, J. E., \& Eriksen, C. W. (1973). Selective encoding from multielement visual displays. Perception \& Psychophysics, 14, 217-224.

Coles, M. G. H., Gratton, G., Bashore, T. R., Eriksen, C. W., \& Donchin, E. (1985). A psychophysiological investigation of the continuous flow model of human information processing. Journal of Experimental Psychology: Human Perception and Performance, $11,529-553$

Cunningham, H. A. (1989). Aiming error under transformed spatial mappings suggests a structure for visual-motor maps. Journal of Experimental Psychology: Human Perception and Performance, 15, 493-506.

Cunningham, H. A., \& Pavel, M. (1991). Target axis effects under transformed visual-motor mappings. In S. R. Ellis, M. K. Kaiser, \& A. J. Grunwald (Eds.), Pictorial communication in virtual and real environments (pp. 283-294). Bristol, PA: Taylor \& Francis.

Dassonville, P., Lewis, S. M., Foster, H. E., \& Ashe J. (1999). Choice and stimulus-response compatibility affect duration of response selection. Cognitive Brain Research, 7, 235-240.

Deininger, R. L., \& Fitts, P. M. (1955). Stimulus-response compatibility, information theory, and perceptual-motor performance. In $\mathrm{H}$. Quatler (Ed.), Information theory in psychology: Problems and methods (pp. 316-341). Glencoe, IL: The Free Press.

Donders, F. C. (1969). On the speed of mental processes. Acta Psychologica, 30, 412-431. (Translation of original article; original work published 1868)

Eriksen, B. A., \& Eriksen, C. W. (1974). Effects of noise letters upon the identification of a target letter in a nonsearch task. Perception \& Psychophysics, 16, 143-149.

Eriksen, C. W., \& Eriksen, B. A. (1979). Target redundancy in visual search: Do repetitions of the target within the display impair processing? Perception \& Psychophysics, 26, 195-205.

Eriksen, C. W., \& Hoffman, J. E. (1972a). Some characteristics of selective attention in visual perception determined by vocal reaction time. Perception \& Psychophysics, 11, 169-171.

Eriksen, C. W., \& Hoffman, J. E. (1972b). Temporal and spatial characteristics of selective encoding from visual displays. Perception \& Psychophysics, 12, 201-204.

Eriksen, C. W., \& Hoffman, J. E. (1973). The extent of processing of noise elements during selective encoding from visual displays. Perception \& Psychophysics, 14, 155-160.

Eriksen, C. W., \& St. James, J. D. (1986). Visual attention within and around the field of focal attention: A zoom lens model. Perception \& Psychophysics, 40, 225-240.

Fitts, P. M. (1954). The information capacity of the human motor system in controlling the amplitude of movement. Journal of Experimental Psychology, 47, 381-391.

Fitts, P. M. (1964). Perceptual-motor skill learning. In A. W. Melton (Ed.), Categories of human learning (pp. 243-285). New York, NY: Academic Press.

Fitts, P. M., \& Deininger, R. L. (1954). S-R compatibility: Correspondence among paired elements within stimulus and response codes. Journal of Experimental Psychology, 48, 483-492.

Fitts, P. M., \& Seeger, C. M. (1953). S-R compatibility: Spatial characteristics of stimulus and response codes. Journal of Experimental Psychology, 46, 199-210.

Garner, W. R., Hake, H. W., \& Eriksen, C. W. (1956). Operationism and the concept of perception. Psychological Review, 63, 149-159. 
Gratton, G., Coles, M. G., Sirevaag, E. J., Eriksen, C. W., \& Donchin, E. (1988). Pre-and poststimulus activation of response channels: A psychophysiological analysis. Journal of Experimental Psychology: Human Perception and Performance, 14, 331-344.

Healy, A. F., \& Bourne, L. E., Jr. (2012). Training cognition: Optimizing efficiency, durability, and generalizability. New York, NY: Psychology Press.

Healy, A. F., Wohldmann, E. L., \& Bourne, L. E., Jr. (2011). How does practice with a reversed mouse influence subsequent speeded aiming performance? A test of global inhibition. Journal of Cognitive Psychology, 23, 559-573

Healy, A. F., Wohldmann, E. L., Sutton, E. M., \& Bourne, L. E., Jr. (2006). Specificity effects in training and transfer of speeded responses. Journal of Experimental Psychology: Learning, Memory, and Cognition, 32, 534-546.

Hick, W. E. (1952). On the rate of gain of information. Quarterly Journal of Experimental Psychology, 4, 11-26.

Hommel, B., Brown, S. B. R. E., \& Nattkemper, D. (2016). Human action control: From intentions to movements. Berlin, Germany: Springer.

Hyman, R. (1953). Stimulus information as a determinant of RT. Journal of Experimental Psychology, 45, 188-196.

Janczyk, M., \& Lerche, V. (2019). A diffusion model analysis of the response-effect compatibility effect. Journal of Experimental Psychology: General, 148, 237-251.

Keele, S. W. (1968). Movement control in skilled motor performance. Psychological Bulletin, 70, 387-403.

Kieras, D. (2009). Why EPIC was wrong about motor feature programming. In A. Howes, D. Peebles, \& R. Cooper (Eds.), 9th International Conference on Cognitive Modeling - ICCM2009, Manchester, UK.

Koch, I., Keller, P., \& Prinz, W. (2004). The ideomotor approach to action control: Implications for skilled performance. Journal of Sport and Exercise Psychology, 2, 362-375.

Kornblum, S., Hasbroucq, T., \& Osman, A. (1990). Dimensional overlap: Cognitive basis for stimulus-response compatibility-A model and taxonomy. Psychological Review, 97, 253-270.

Kunde, W. (2001). Response-effect compatibility in manual choice reaction tasks. Journal of Experimental Psychology: Human Perception and Performance, 27, 387-394.

Kunde, W., Koch, I., \& Hoffmann, J. (2004). Anticipated action effects affect the selection, initiation, and execution of actions. Quarterly Journal of Experimental Psychology, 57A, 87-106.

Liu, W., Rioul, O., Beaudouin-Lafon, M., \& Guiard, Y. (2017). Information-theoretic analysis of human performance for command selection. In IFIP Conference on Human-Computer Interaction (pp. 515-524). Cham, Switzerland: Springer.

Logan, G. D., \& Crump, M. J. C. (2011). Hierarchical control of cognitive processes: The case for skilled typewriting. In B. Ross (Ed.), The psychology of learning and motivation (Vol. 54, pp. 1-27). Burlington, MA: Academic Press.

Luo, C., \& Proctor, R. W. (2020). The location-based Simon effect: Reliability of ex-Gaussian analysis. Memory \& Cognition, 48, 4250.

Masson, M. E. (2018). Intentions and actions. Canadian Journal of Experimental Psychology, 72, 219-228.

Mattler, U. (2005). Flanker effects on motor output and the late-level response activation hypothesis. Quarterly Journal of Experimental Psychology, 58, 577-601.

Meyer, D. E., Abrams, R. A., Kornblum, S., Wright, C. E., \& Smith, J. E. K. (1988). Optimality in human motor performance: Ideal control of rapid aimed movements. Psychological Review, 95, 340-370.

Meyer, D. E., \& Kieras, D. E. (1997). A computational theory of executive cognitive processes and multiple-task performance: 1. Basic mechanisms. Psychological Review, 104, 3-65.
Morin, R. E., \& Grant, D. A. (1955). Learning and performance of a keypressing task as a function of the degree of spatial stimulus-response correspondence, Journal of Experimental Psychology, 49, 39-47.

Murata, A. (1999). Extending effective target width in Fitts' law to a twodimensional pointing task. International Journal of HumanComputer Interaction, 11, 137-152.

Pappas, Z. (2014). Dissociating Simon and affordance compatibility effects: Silhouettes and photographs. Cognition, 133, 716-728.

Pauli, P., Braun, C., Wiech, K., Birbaumer, N., \& Bourne, L. E., Jr. (2005). Brain processes associated with target finding. Cognitive Brain Research, 25, 926-935.

Proctor, R. W., \& Cho, Y. S. (2006). Polarity correspondence: A general principle for performance of speeded binary classification tasks. Psychological Bulletin, 132, 416-442.

Proctor, R. W., \& Miles, J. D. (2014). Does the concept of affordance add anything to explanations of stimulus-response compatibility effects? In B. H. Ross (Ed.), The psychology of learning and motivation (Vol. 60, pp. 227-266). Burlington, MA: Academic Press.

Proctor, R. W., \& Schneider, D. W. (2018). Hick's law for choice reaction time: A review. Quarterly Journal of Experimental Psychology, 71, $1281-1299$.

Proctor, R. W., \& Vu, K.-P. L. (2006). Stimulus-response compatibility principles: Data, theory, and application. Boca Raton, FL: CRC Press.

Proctor, R. W., \& Vu, K.-P. L. (2016). Principles for designing interfaces compatible with human information processing. International Journal of Human-Computer Interaction, 32, 2-22.

Roy, Q., Guiard, Y., Bailly, G., Lecolinet, É., \& Rioul, O. (2015, September). Glass+ skin: An empirical evaluation of the added value of finger identification to basic single-touch interaction on touch screens. In J. Abascal et al. (Eds.), Human-Computer InteractionINTERACT 2015 (pp. 55-71). Cham, Switzerland: Springer.

Sherwood, D. E., Lohse, K. R., \& Healy, A. F. (2020). The effect of an external and internal focus of attention on dual-task performance. Journal of Experimental Psychology: Human Perception and Performance, 46, 91-104.

Shin, Y. K., Proctor, R. W., \& Capaldi, E. J. (2010). A review of contemporary ideomotor theory. Psychological Bulletin, 136, 943-974.

Sternberg, S. (1969). The discovery of processing stages: Extensions of Donders' method. Acta Psychologica, 30, 276-315.

Thorndike, E. L. (1906). The principles of teaching: Based on psychology. New York, NY: A. G. Seiler.

Tucker, M., \& Ellis, R. (1998). On the relations between seen objects and components of potential actions. Journal of Experimental Psychology: Human Perception and Performance, 24, 830-846.

Umiltá, C., \& Nicoletti, R. (1990). Spatial stimulus-response compatibility. In R. W. Proctor \& T. G. Reeve (Eds.), Stimulus-response compatibility: An integrated perspective (pp. 89-116). Amsterdam, The Netherlands: North-Holland.

Verbruggen, F., Liefooghe, B., Notebaert, W., \& Vandierendonck, A. (2005). Effects of stimulus-stimulus compatibility and stimulusresponse compatibility on response inhibition. Acta Psychologica, 120, 307-326.

Wohldmann, E. L., \& Healy, A. F. (2010). Exploring specificity of speeded aiming movements: Examining different measures of transfer. Memory \& Cognition, 38, 344-355.

Wohldmann, E. L., Healy, A. F., \& Bourne, L. E., Jr. (2008). Global inhibition and midcourse corrections in speeded aiming. Memory \& Cognition, 36, 1228-1235.

Wright, C. E., Marino, V. F., Belovsky, S. A., \& Chubb, C. (2007). Visually guided, aimed movements can be unaffected by stimulus-response uncertainty. Experimental Brain Research, 179, 475-496.

Wright, C. E., Marino, V. F., Chubb, C., \& Mann, D. (2019). A model of the uncertainty effects in choice reaction time that includes a major 
contribution from effector selection. Psychological Review, 126, $550-577$.

Wright, C. E., Marino, V. F., Chubb, C., \& Rose, K. A. (2011). Exploring attention-based explanations for some violations of Hick's law for aimed movements. Attention, Perception, \& Psychophysics, 73, 854-871.

Wühr, P., \& Heuer, H. (2017). Response preparation, response conflict, and the effects of irrelevant flanker stimuli. Advances in Cognitive Psychology, 13, 70-82.

Wulf, G., \& Lewthwaite, R. (2016). Optimizing performance through intrinsic motivation and attention for learning: The OPTIMAL theory of motor learning. Psychonomic Bulletin \& Review, 23, $1382-1414$.

Xiong, A., \& Proctor, R. W. (2018). The role of task space in action control: Evidence from research on instructions. In K. D. Federmeier (Ed.), The psychology of learning and motivation (Vol. 69, pp. 325-364). Cambridge, MA: Academic Press.

Publisher's note Springer Nature remains neutral with regard to jurisdictional claims in published maps and institutional affiliations. 